\title{
3D-FBK Pixel sensors: recent beam tests results with irradiated devices
}

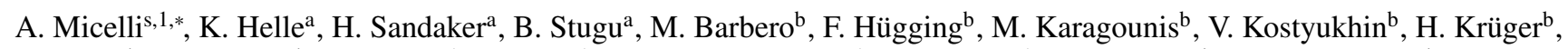
J-W Tsung ${ }^{\mathrm{b}}$, N. Wermes ${ }^{\mathrm{b}}$, M. Capua ${ }^{1}$, S. Fazio ${ }^{1}$, A. Mastroberardino ${ }^{1}$, G. Susinno ${ }^{1}$, C. Gallrapp ${ }^{\mathrm{d}}$, B. Di Girolamo ${ }^{\mathrm{d}}$, D. Dobos $^{\mathrm{d}}$, A. La Rosa ${ }^{d}$, H. Pernegger ${ }^{\mathrm{d}}$, S. Roe ${ }^{\mathrm{d}}$, T Slavicek $^{1}$, S. Pospisil ${ }^{1}$, K. Jakobs ${ }^{\mathrm{g}}$, M. Köhler ${ }^{\mathrm{g}}$, U. Parzefall ${ }^{\mathrm{g}}$, G. Darbo ${ }^{\mathrm{h}}$, G. Gariano ${ }^{\mathrm{h}}$, C. Gemme ${ }^{1}$, A. Rovani ${ }^{\text {h }}$, E. Ruscino ${ }^{\mathrm{h}}$, C. Butter ${ }^{\mathrm{i}}$, R. Bates ${ }^{\mathrm{i}}$, V. Oshea ${ }^{\mathrm{i}}$, S. Parker ${ }^{1}$, M. Cavalli-Sforza ${ }^{\mathrm{k}}$, S. Grinstein $^{\mathrm{k}}$,

I. Korokolov ${ }^{\mathrm{k}}$, C. Pradilla ${ }^{\mathrm{k}}$, K. Einsweiler ${ }^{\mathrm{l}}$, M. Garcia-Sciveres ${ }^{1}$, M. Borri $^{\mathrm{m}}$, C. Da Viàm ${ }^{\mathrm{m}}$ J. Freestone $^{\mathrm{m}}$, S. Kolya ${ }^{\mathrm{m}}$, C. H.Lai ${ }^{\mathrm{m}}$,

C. Nellist ${ }^{\mathrm{m}}$, J. Pater ${ }^{\mathrm{m}}$, R. Thompson ${ }^{\mathrm{m}}$, S. J. Watts ${ }^{\mathrm{m}}$, M. Hoeferkamp ${ }^{\mathrm{n}}$, S. Seidel $^{\mathrm{n}}$, E. Bolle ${ }^{\mathrm{o}}$, H. Gjersdal $^{\mathrm{o}}$, K-N Sjoebaek ${ }^{\mathrm{o}}$,

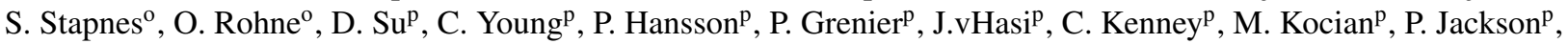

D. Silverstein ${ }^{\mathrm{p}}$, H. Davetak ${ }^{\mathrm{q}}$, B. DeWilde ${ }^{\mathrm{q}}$, D. Tsybychev ${ }^{\mathrm{q}}$, G-F Dalla Betta ${ }^{\mathrm{r}}$, P. Gabos ${ }^{\mathrm{r}}$, M. Povoli ${ }^{\mathrm{r}}$, M. Cobal ${ }^{\mathrm{s}}$, M-P Giordani ${ }^{\mathrm{s}}$,

L. Selmi ${ }^{\mathrm{s}}$, A. Cristofoli ${ }^{\mathrm{s}}$, D. Esseni ${ }^{\mathrm{s}}$, P. Palestri ${ }^{\mathrm{s}}$, C. Fleta ${ }^{\mathrm{t}}$, M. Lozano ${ }^{\mathrm{t}}$, G. Pellegrini ${ }^{\mathrm{t}}$, M. Boscardin ${ }^{\mathrm{u}}$, A. Bagolini ${ }^{\mathrm{u}}$, C. Piemonte ${ }^{\mathrm{u}}$, S. Ronchin ${ }^{\mathrm{u}}$, N. Zorzi ${ }^{\mathrm{u}}$, T-E. Hansen ${ }^{\mathrm{v}}$, T. Hansen ${ }^{\mathrm{v}}$, A. Kok ${ }^{\mathrm{v}}$, N. Lietaer ${ }^{\mathrm{v}}$, J. Kalliopuska ${ }^{\mathrm{x}}$, A. Oja ${ }^{\mathrm{x}}$

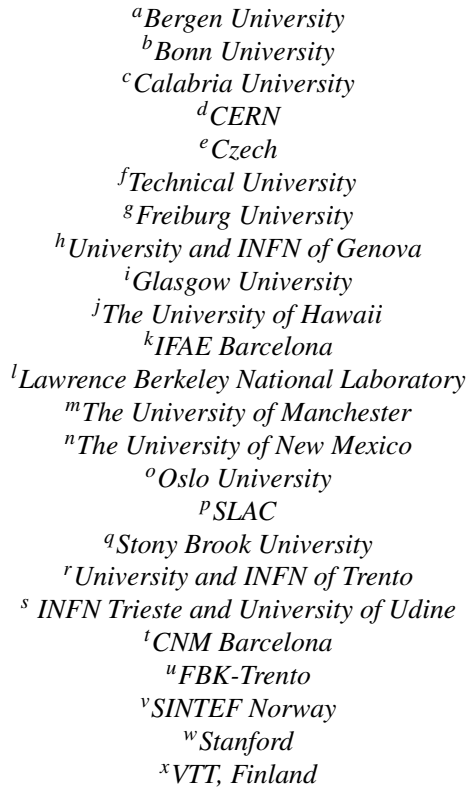

\begin{abstract}
The Pixel detector is the innermost part of the ATLAS experiment tracking device at the Large Hadron Collider (LHC), and plays a key role in the reconstruction of the primary and secondary vertices of short-lived particles. To cope with the high level of radiation produced during the collider operation, it is planned to add to the present three layers of silicon pixel sensors which constitute the Pixel Detector, an additional layer (Insertable B-Layer, or IBL) of sensors. 3D silicon sensors are one of the technologies which are under study for the IBL. 3D silicon technology is an innovative combination of very-large-scale integration (VLSI) and MicroElectro-Mechanical-Systems (MEMS) where electrodes are fabricated inside the silicon bulk instead of being implanted on the wafer surfaces. 3D sensors, with electrodes fully or partially penetrating the silicon substrate, are currently fabricated at different processing facilities in Europe and USA. This paper reports on the 2010 June beam test results for irradiated 3D devices produced at FBK (Trento, Italy). The performance of these devices, all bump-bonded with the ATLAS pixel FE-I3 readout chip, is compared to that observed before irradiation in a previous beam test.
\end{abstract}

Keywords:

Silicon sensors, 3D sensors, radiation detectors, ATLAS upgrade, SLHC

\section{Introduction}

${ }^{*}$ Corresponding author. Tel.: +41227672671.

Email address: andrea.micelli@cern.ch (A. Micelli)
The ATLAS Pixel detector [1] is the innermost layer of the tracking system the ATLAS experiment at the LHC. It is charac- 
terized by a high level of granularity and it can provide a set of precise measurements at a very short distance $(5-15 \mathrm{~cm})$ from to the Interaction Point (IP). For these reasons, it plays a key role in the reconstruction of primary and secondary vertices of short-lived particles.

Being so close to the IP, the Pixel detector requires an excellent radiation hardness, mechanical and thermal robustness, and a long-term stability. In addition to all this, a low material budget is required. The issue of radiation hardness is particularly critical: after just three years of LHC operation at its nominal instantaneous luminosity of $10^{34} \mathrm{~cm}^{-2} \mathrm{~s}^{-1}$, the Inner layer of the Pixel detector (B-layer) will have accumulated a fluence of $\simeq 10^{15} \mathrm{n}_{e q}$. $/ \mathrm{cm}^{2}$, which will significantly degrade its performance. To mantain - and even improve - this last one, the ATLAS Collaboration has decided to insert into the layer which presently is the innermost one, an additional layer of sensors, called Insertable B-Layer (IBL) [2]. Looking even more into the future, the ATLAS experiment will require a brand new Inner Tracking Detector to cope with the upgrade of the LHC by one order of magnitude in luminosity beyond the design value, expected in the proposed High Luminosity LHC (HLLHC) project. The 3D design [3] for the silicon pixel sensors seems to be a good candidate for both scenarios.

To this purpose, the 3D ATLAS R\&D Collaboration has been started [4], which includes several institutes and five processing facilities from Europe and USA. In 2009 three beam tests have been performed, and results have been previously reported in [5], [6], [7]. This paper is concerned with the first beam test of 2010, describing the setup and selected results for irradiated 3D sensors fabricated at Fondazione Bruno Kessler (FBK) in Trento, Italy [8]. The peculiar structure of the considered sensors and results from previous beam tests and laboratory tests are recalled in order to gain insight into the observed charge collection properties and to validate the data analysis.

\section{3D Sensors Design and Technology}

The 3D architecture was originally proposed by Parker et al. more then ten years ago [3]. The electrodes in 3D detectors are fabricated by etching holes in a silicon substrate, typically with Deep Reactive Ion Etching (DRIE), then filling them with polysilicon [9]. This makes 3D sensors considerably more complicated to produce than traditional planar sensors. Different designs are currently under study: the Full-3D sensors fabricated at Stanford [10] and in parallel at SINTEF [11] with fully penetrating electrodes, and the modified-3D sensors by FBK and by the Centro National de Microelectronica (CNM) of Barcelona, Spain [12] in which the electrodes do not penetrate the entire substrate thickness.

Compared to traditional planar sensors, for which electrodes are processed on the wafer surface, both 3D designs have several important distinguishing features: $(i)$ higher average electric field between the electrodes, (ii) a shorter collection path, implying a lower full depletion voltage, and (iii) advantages in extreme radiation environments because the charge carrier drift-length is decoupled from the sensor thickness [13] and therefore the signal efficiency depends only on the geometrical

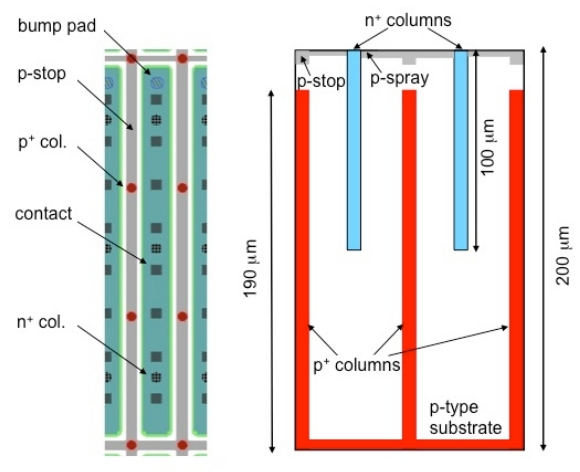

Figure 1: Schematic cross-section of the Double-Sided Double-Type modified3D sensors used for this study, where the $n^{+}$and $p^{+}$electrodes are only partially overlapping.

inter-electrode distance between the $\mathrm{p}^{+}$and $\mathrm{n}^{+}$electrodes. Additionally, Full-3D sensors also feature active edges, which can reduce the dead areas at the sensor periphery to a few $\mu \mathrm{m}$ [14], whereas in modified-3D sensors a slim edge of a few hundreds of $\mu \mathrm{m}$ can be achieved [15]. Among the currently considered modified-3D sensors, in this paper we focus on those fabricated at FBK, which have been used in the 2010 beam test. .

\section{1. $3 D-D D T C$}

FBK and the Italian National Institute for Nuclear Physics (INFN) have collaborated since 2004 to develop modified 3D sensors, aimed at a simplification of the manufacturing technology with respect to the original Full-3D design. Historically, as a first step, the 3D-STC (Single Type-Column) was proposed [16], having columnar electrodes of one doping type only $\left(n^{+}\right)$ and not penetrating all the way through the substrate (on the back-side a uniform $p^{+}$ohmic contact is present). The second step was the 3D Double Sided Double Type Columns (DDTC) detectors, which are aimed at performance enhancement while maintaining a reasonably simple fabrication process. These detectors are being considered as an alternative to Full-3D detectors in the ATLAS Pixel Detector upgrade for the sLHC, possibly allowing for a reduction of process complexity and cost. A similar approach is being independently developed by CNM [17].

In these sensors, columns of one doping type are etched from the front-side of the device, and columns of the other type are etched from the back side (see Fig. 1). Neither set of columns passes through the full thickness of the substrate. Column etching is stopped at short distance (d, ideally not exceeding a few tens of $\mu \mathrm{m}$ ) from the opposite surface. Frontside columns are used for readout (junction columns), whereas back-side columns are all connected together and used to bias the sensor. In 3D-DDTC sensors made at FBK, columns have nominal diameter of $10 \mu \mathrm{m}$ for both doping types and are not filled with poly-Si. Besides the ohmic columns, the entire backside is $\mathrm{p}+$ doped and coated with metal. Having the bias contact on the back side, rather than on the front-side as in the original 3D design, also eases the detector assembly with standard readout electronics. The fabrication technology for FBK 3D- 
DDTC sensors is detailed in [18] for the first prototypes that were fabricated on n-type substrates. The only differences for the sensors considered in this paper are: (i) the substrate type, (ii) the inverted doping of the columns and related surface regions, and (iii) the additional steps for the p-spray/p-stop implantations on the front surface. Different layout versions of FBK sensors have been studied, featuring two (2E), three (3E) and four (4E) equally spaced electrodes per pixel (see Fig. 2). The pixel design matches that of the ATLAS FE-I3 readout chip [19], with a 160x18 array of pixels of $400 \times 50 \mu \mathrm{m}^{2}$ area. More details on the sensor design can be found in [20].

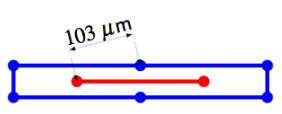

(a) $2 \mathrm{E}$

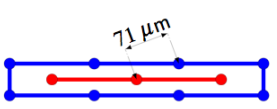

(b) $3 \mathrm{E}$

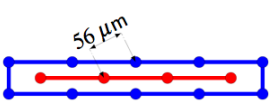

(c) $4 \mathrm{E}$
Figure 2: Schematic view of the different 3D layout configurations with 2, 3, and 4 read-out electrodes per pixel.

\section{Radiation damage of silicon detectors}

Since 3D detectors are being investigated to be possibly used for the IBL, it has to be proved that they are resistant to the high level of radiation expected in the next years. Sensors need to be qualified for radiation of $300 \mathrm{Mrad}$ or fluence of $5 \times 10^{15}$ 1-MeV equivalent neutrons $\left(\mathrm{n}_{e q}\right)$ per $\mathrm{cm}^{2}$.

The radiation damage is divided into two main effects: bulk and surface damage. The first is caused by the displacement of crystal atoms while the second includes all effects taking place in the dielectric layers covering the sensors. The most important surface effect is the charge density increase in the oxide passivation layer, which saturates after some $\mathrm{kGy}$ to values of about $3 \times 10^{12} \mathrm{~cm}^{-2}$. At high hadron fluences, bulk damage becomes the most important. Its main consequences for detectors are: $(i)$ the increase of the leakage current, proportional to the fluence. This leads to an increased power dissipation, which heats the sensor, and higher temperature implies higher leakage current and therefore larger dissipated power. The result is a positive feedback system that may quickly diverge (thermal runaway), unless prevented by proper cooling; (ii) the increase of the effective doping concentration and subsequent increase of the full-depletion voltage; (iii) charge trapping. Usually traps are mostly unoccupied due to the lack of free charge carriers, and can hold or trap part of the signal charge for a time longer than the charge collection time and consequently reduce the signal height .

Preliminary results about the first step of the sensor irradiation program, corresponding to a fluence of $1 \times 10^{15} \mathrm{n}_{e q} / \mathrm{cm}^{2}$, are presented in this paper.

\section{Test Beam Studies}

\subsection{Test Beam Setup}

During the first part of 2010, 3D silicon sensors were tested and characterized in data-taking at Cern SPS North Area. The
June test beam used the $\mathrm{H} 6$ beam line with a $120 \mathrm{GeV} / \mathrm{c} \pi^{+}$ beam) and the European Detector (EUDET) beam telescope [21]; the setup is shown schematically in Fig. 3.

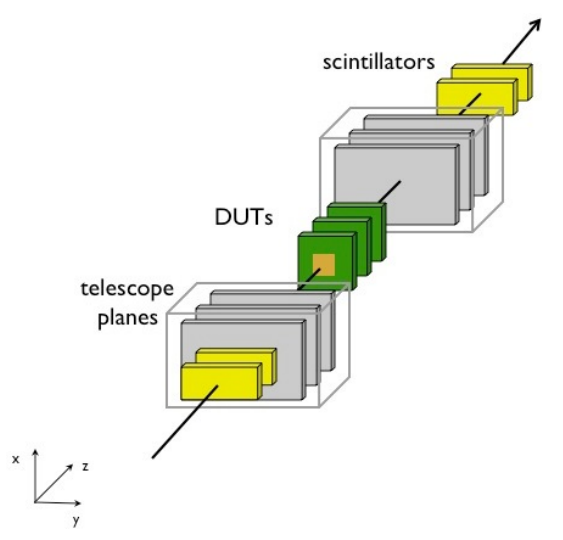

Figure 3: Schematic view of the EUDET test beam setup.

The EUDET Pixel Telescope, consists of three planes for each side separated by about $40 \mathrm{~cm}$, distributed before and after the Devices Under Tests (DUTs). The core of the telescope is the Mimosa26 pixel sensor with a pitch of $18.5 \mu \mathrm{m}$. Each sensor has 576x1152 pixels covering an active area of 10.6x21.2 $\mathrm{mm}^{2}$. Triggering was achieved by the use of two up- and two down-stream sets of $1 \times 2 \mathrm{~cm}^{2}$ scintillators positioned at $90^{\circ}$ with respect to each other. DUTs were located between the two telescope arms and were mounted on remotely controlled rotating stages. The EUDET tracking resolution has been estimated to be about $3 \mu \mathrm{m}$. The purpose of this telescope was to characterize the sensors performance after irradiation at different tilted angles (from $-25^{\circ}$ to $+25^{\circ}$ ) with the usual figures of merit, i.e.: tracks efficiency, charge sharing.

\subsection{Devices Under Test}

Two devices under test were used for the June test beam period along with $250 \mu \mathrm{m}$ thick ATLAS planar pixel sensor as a reference [22] The two DUTs are both 3D-DDTC sensors fabricated at FBK and belonging to the production batch called 3DDTC-2. Specific to this batch is the strongly asymmetric depth of columnar electrodes: ohmic columns are almost as deep as the substrate $(190 \mu \mathrm{m}$ vs $200 \mu \mathrm{m})$, whereas junction columns are about $100 \mu \mathrm{m}$ deep (see Fig. 1). This non optimized configuration was the result of the need to reduce the etching time of junction columns due to excessive wafer breakage during this step [23]. The two DUTs have been irradiated at a fluence of $10^{15} \mathrm{n}_{e q} / \mathrm{cm}^{2}$ with protons and neutrons, respectively. The Bonn group managed the irradiation with $27-\mathrm{MeV}$ protons at the Karlruhe facility [24], whereas the neutron irradiation was performed at the JSI neutron reactor in Ljubljana [25]. Both 3D sensors are of the $3 \mathrm{E}$ type (corresponding to an inter-electrode pitch of $71 \mu \mathrm{m}$ ). All three tested sensors were bump-bonded to the ATLAS Front-End chip I3 (FE-I3) [19]. Bump bonding was performed at SELEX [26] for 3D sensors and at IZM [27] for the planar reference sensor. The FE-I3 consists of 2880 readout cells (a matrix of 160 rows $\times 18$ columns) of $50 \times 400 \mu \mathrm{m}^{2}$. 
It provides pixel charge measurement through the digital timeover-threshold (TOT) measured in units of LHC bunch crossings ( $25 \mathrm{~ns}$ ). The charge threshold and TOT-to-charge conversion have been tuned to each individual pixel to $3200 \mathrm{e}^{-}$and 60 TOT, respectively, corresponding to a total deposited charge of $20000 \mathrm{e}^{-}$. During measurements, DUTs were cooled at $-20^{\circ} \mathrm{C}$. The two 3D sensors were reverse biased at $80 \mathrm{~V}$, a voltage for which lateral depletion is ensured in those regions where the columnar electrodes overlap, whereas the bottom of the substrate is not fully depleted. The voltage could not be increased further due to onset of breakdown effects.

\section{Results}

\subsection{Tracking Efficiency}

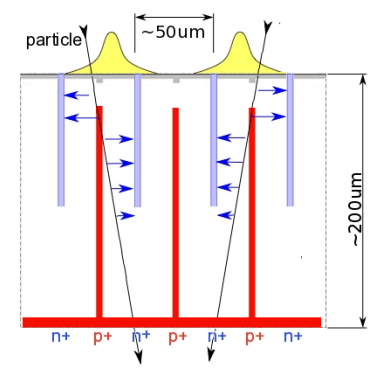

(a)

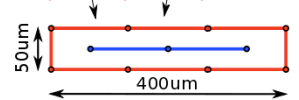

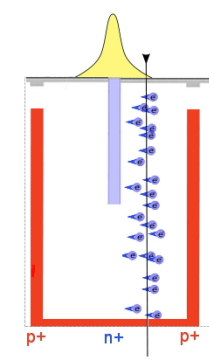

(b)

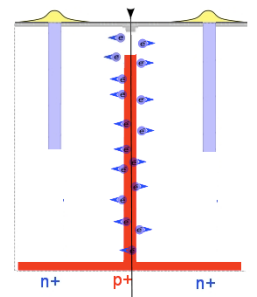

(c)
Figure 4: Schematic views of the passage charge particle: (a) with tilted beam (two sensor cells visible); normal incidence of the beam: two possible track paths are shown: (b) through the bulk and through a bias electrode (c).

In full-3D sensors, charge collection from particle tracks passing through the silicon bulk is well understood, whereas for tracks passing through the region in and very close to $3 \mathrm{D}$ electrodes the collection process is more complicate, and currently still under study. Many parameters normally affect the signals expected from such tracks, among them the exact diameter of the electrodes, the doping technique for poly-Si and the doping profile depths in silicon. Moreover, systematic uncertainties arise from the alignment of the sensors in the beam telescope, as well as the incident angle of the beam itself. In the considered modified-3D sensors, columns are hollow, so do not contribute any signal. However, it should be stressed that the junction columns are only about $100 \mu \mathrm{m}$ deep: on one hand, this can provide non negligible signals for tracks passing through the electrodes, due to the contributions from the substrate region below the column tips; on the other hand, it should be noted that a low electric field is present through a significant fraction of the active volume at the bottom of the devices, so that trapping effects can become more severe after irradiation.

Clearly, tracking efficiency is of fundamental importance in the study and understanding of 3D sensors. Fig. 5 shows results relevant to the two irradiated sensors for different tilting angles. Results are also summarized in Table 1 and compared to those relevant to a non irradiated $3 \mathrm{E}$ sensor from the same batch as those considered in this study, which was tested in a beam test in June 2009 [6].
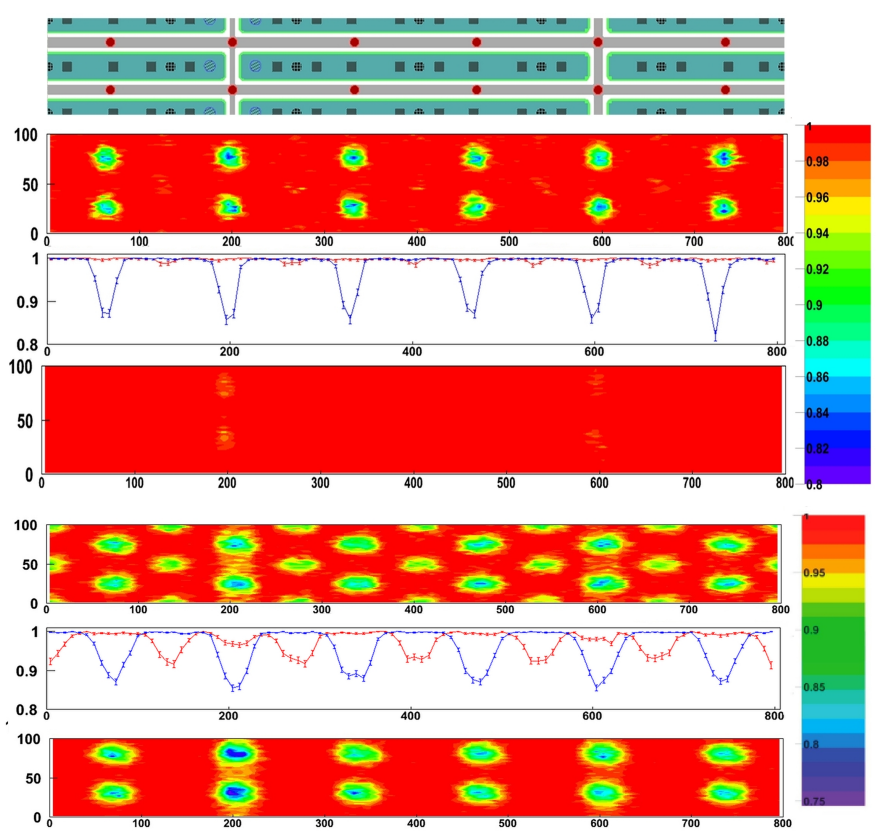

Figure 5: Efficiency maps reconstructed from the Eudet data for the 3D sensor after proton and neutron irradiation. From top to bottom: (a) mask detail centered on one cell and extending to half a cell in both directions; (b) two-dimensional efficiency map at $0^{\circ}$ for the proton irradiated sensor; (c) onedimensional efficiency projections of (b) along planes including the read-out (blue curve) and bias (red curve) electrode regions at $0^{\circ}$; (d) same as (b) at $15^{\circ}$; (e) two-dimensional efficiency map at $0^{\circ}$ for the neutron irradiated sensor; (f) one-dimensional efficiency projections of (e) along planes including the readout (blue curve) and bias (red curve) electrode regions at $0^{\circ} ;(\mathrm{g})$ same as (e) at $15^{\circ}$.

In the proton irradiated sensor, at $0^{\circ}$ (slice b), a small efficiency drop is visible for the read-out electrodes, and a more pronounced one for the bias electrodes and surrounding regions. Details can be also appreciated in slice c, showing the $1 \mathrm{~d}$ projections of data along planes passing through the read-out electrodes (red) and bias electrodes (blue). Observed results are not surprising, given the large difference in the column depths and the higher electric field close to the read-out electrodes. At $15^{\circ}$ (slice $\mathrm{d}$ ), that could be the case for the IBL application, an almost $100 \%$ efficiency is recovered everywhere but in the four bias columns at the corners of a pixel, most likely due to charge sharing effects that reduce the collected charge and so cause some hits to be missed.

In the neutron irradiated sensor, at $0^{\circ}$ (slices e and $\mathrm{f}$ ), the regions with lower efficiency associated to the electrodes become wider. At $15^{\circ}$ (slice g), full efficiency is recovered for the read-out electrode regions, whereas for all bias electrodes and surrounding regions the efficiency remains significantly lower, the worst cases being still represented by the four electrodes at the corners of the pixel. The overall efficiency for the different cases is reported in Table 1 and compared to the pre-irradiation case. It can be concluded that the efficiency is not significantly degraded in the proton irradiated sensor, whereas some degradation is observed after neutron irradiation. The difference between results for protons and neutrons is likely to be attributed to the fact that neutrons have a higher introduction rate of sta- 

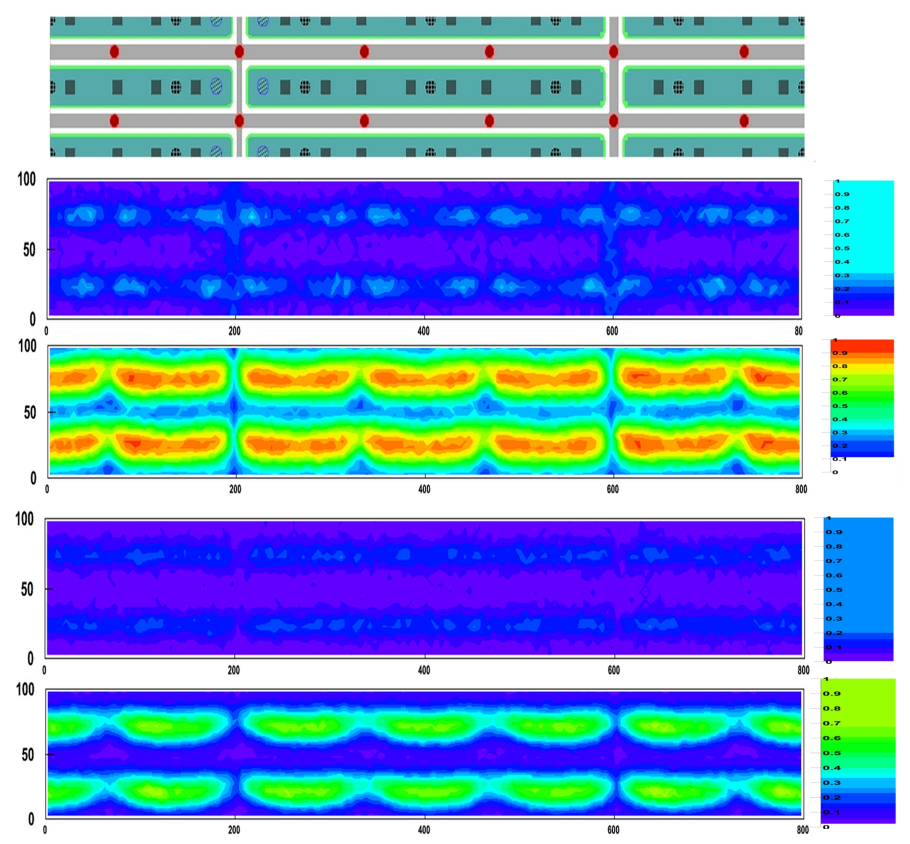

Figure 6: Charge sharing maps in three 3D sensors. From top to bottom: (a) mask detail centered on one cell and extending to half a cell in both directions; (b) proton irradiated sample at normal incidence (c) same as (b) at $10^{\circ}$; (d) and (e) same as (b) and (c) for the neutron irradiated samples at $0^{\circ}$ and at $15^{\circ}$.

ble acceptor defects with respect to protons [28]. As a result, at the $80 \mathrm{~V}$ bias used for the beam test, insufficient depletion could decrease the efficiency of some critical regions of the sensor, i.e., below read-out column tips and close to the bias columns. These aspects are currently being investigated with the aid of TCAD simulations.

\subsection{Charge Sharing}

The sharing of charge between two neighboring pixel cells has been studied. Here it is defined as the ratio of the number of tracks with more than one hit over the total number of tracks: $N_{\text {tracks }}(>1$ hit $) / N_{\text {tracks }}($ all $)$. This parameter is an important feature of pixel detectors because, in case of charge sharing, only a fraction of the total deposited charge is available in each cell, making it more difficult for the readout circuit to cross the threshold in each pixel. For highly irradiated sensors, where a large fraction of the charge is lost due to the trapping, charge sharing is therefore expected to increase the inefficiency of the detector. Due to their peculiar structure, which provides a sort of self-shielding effect in each cell, in Full-3D sensors charge sharing between adjacent electrodes is considerably reduced as compared to planar sensors. However, in the considered modified-3D sensors, this feature can not be fully exploited due to the non optimized column depth. This effect is of course more pronounced in case sensors are tilted, this configuration easing the split of the charge between adjacent cells.

Fig. 6 reports the maps of charge sharing for the two irradiated sensors at tilt $0^{\circ}$ and $15^{\circ}$ (note the different color coding for protons and neutrons). Results are also summarized in Table 1 and Fig. 9 shows the probability of charge sharing as function of track position within the pixel cell. For both the pro- ton and neutron irradiated sensors, charge sharing at $0^{\circ}$ (slices a and c) is pretty small, and is only slightly increased at the cell boundaries, particularly along the long pixel edge side of the pixels, where the low field regions are wider. As expected, charge sharing is considerably larger at $15^{\circ}$ (slices $b$ and $d$ ) for both sensors. Notably, the overall charge sharing for protons is found to be about two times larger than for neutrons. From 6, charge sharing is indeed pretty similar for neutrons and protons at the pixel boundaries, whereas it is markedly different for central regions: values for the proton irradiated sample are similar to those before irradiation (see Table 1), whereas charge sharing is strongly attenuated in the neutron irradiated sample. The reason for this behavior is not fully understood and will be further investigated in future beam tests. A naive explanation, supported by the results in terms of efficiency, is based on the fact that, due to incomplete depletion of the active volume, charge generated in the central regions of a pixel can more likely get trapped rather than being collected by the adjacent pixels. This hypothesis is currently being checked with the aid of TCAD simulations.

\subsection{Cluster size}
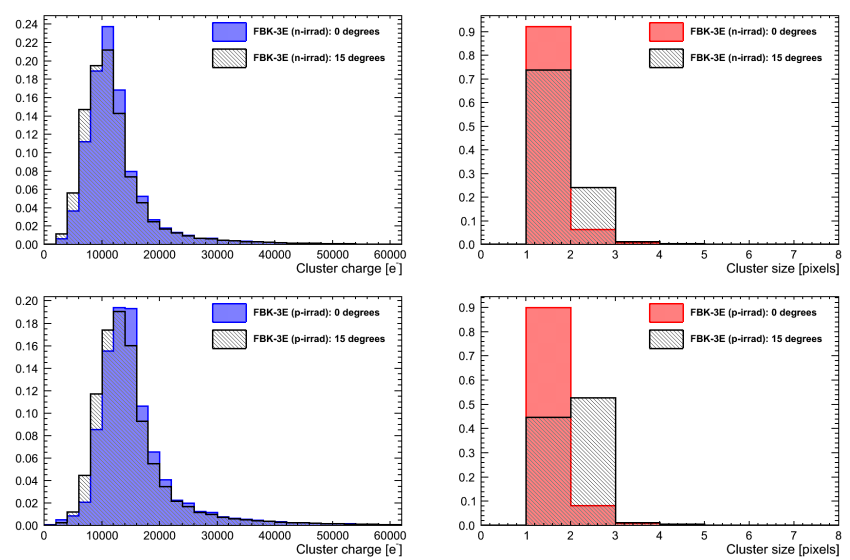

Figure 7: Cluster charge and cluster size for FBK irradiated sensors at two different angle settings. Top: proton irradiated sensor. Bottom neutron irradiated sensor.

Fig. 7 shows the recorded cluster charge and cluster size for the two irradiated sensors at two different angle settings. Main data are also summarized in Table 2 . The signal charge in the neutron irradiated sample is $20 \%$ lower than in the proton irradiated one. For the latter, data can be compared to the pulse height distribution obtained in laboratory with $\operatorname{Sr}^{90} \beta$ source setup. Fig. 8 shows the charge distribution, fitted with a Landau convoluted with Gaussian function, which is also shown in the figure. The mean value if quite close to that measured in the beam test.

For the proton irradiated sensor, roughly half of all tracks at tilted incidence have more than one hit per cluster, in agreement with the charge sharing result. On the other hand, for the neutron irradiated sensor the charge collected is lower (closer to the threshold), which is consistent with a slightly reduced effi- 


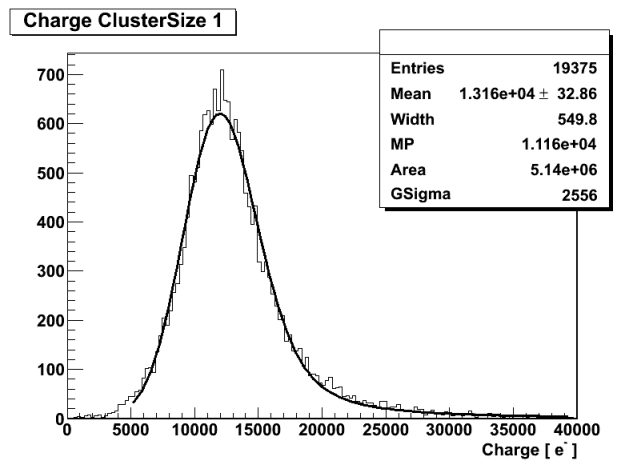

Figure 8: Charge distribution at normal incidence for the proton irradiated sensor biased at $90 \mathrm{~V}$ and measured with $\mathrm{Sr}^{90}$ source setup. Data are relevant to a cluster size equal to 1 .

\begin{tabular}{|l|c|c|c|c|}
\hline & \multicolumn{2}{|c|}{ Hit efficiency [\%] } & \multicolumn{2}{c|}{ Charge sharing [\%] } \\
\hline FBK & $\Phi=0^{\circ}$ & $\Phi=15^{\circ}$ & $\Phi=0^{\circ}$ & $\Phi=15^{\circ}$ \\
\hline n-irrad & 97.6 & 98.1 & 7.0 & 25.2 \\
\hline p-irrad & 99.0 & 99.9 & 9.2 & 54.2 \\
\hline un-irrad & 90.2 & $97.7^{*}$ & 12.2 & 62.1 \\
\hline
\end{tabular}

Table 1: The over-all hit efficiency and the charge sharing are summarized in table. More information for the non irradiated sensor coulb be find in [6]. [*] Note that for the un-irradiated FBK sensor the angle was $14.1^{\circ}$ not $15^{\circ}$.

ciency. The mean luster charge and mean cluster size are listed in Table 2.

\begin{tabular}{|l|c|c|c|c|}
\hline & \multicolumn{2}{|c|}{ Mean cluster charge } & \multicolumn{2}{c|}{ Mean cluster size } \\
\hline FBK & $\Phi=0^{\circ}$ & $\Phi=15^{\circ}$ & $\Phi=0^{\circ}$ & $\Phi=15^{\circ}$ \\
\hline n-irrad & 16.210 & 15,560 & 1.11 & 1.30 \\
\hline p-irrad & 13,010 & 12,560 & 1.14 & 1.60 \\
\hline un-irrad & 16,400 & $15,980^{*}$ & 1.19 & $1.41^{*}$ \\
\hline
\end{tabular}

Table 2: The average cluster charge and size are summarized in table. More information for the non irradiated sensor coulb be find in [6]. [*] Note that for the un-irradiated FBK sensor the angle was $14.1^{\circ}$ not $15^{\circ}$.
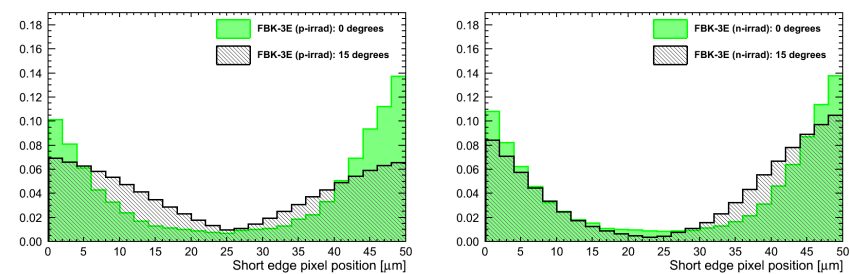

Figure 9: Charge sharing probability in the short direction $(50 \mu \mathrm{m})$ as a fuction of track position within the pixel cell for FBK irradiated sensors. Left: proton irradiated sensor. Right: neutron irradiated sensor.

\section{Conclusions}

3D sensors are a good candidate for the ATLAS Insertable B-Layer. The behavior of 3D-DDTC pixel sensors fabricated at FBK has been reported here. In spite of a non optimized column depth for the electrodes, this modified-3D design present good results in terms of efficiency, charge sharing and radiation hardness, also in agreement with results from laboratory characterization. Comparedto the results obtained on the same sensors before irradiation, only minor degradation was observed after irradiation. Some different features between the proton and neutron irradiated sensors are not fully understood and will be further investigated both in laboratory and next beam tests, for which a magnetic field will be added to the experimental setup. The development of a new, optimized version of modified-3D sensors with passing-through columns is well under way at FBK and the first batch, containing also pixel sensors compatible with the new front-end chip FE-I4, is expected for the end of the year.

\section{References}

[1] The ATLAS Collaboration, JINST 3, (2008) S08003.

[2] M. Capeans et al., CERN-LHCC-2010-013. ATLAS-TDR-019 (2010) 242.

[3] S. I. Parker et al., Nucl. Instrum. Meth. A395 (1997) 328.

[4] C. Da Via, et al., available HYPERLINK http://cern.ch/atlas-highlumi3dsensor, http://cern.ch/atlas-highlumi-3dsensor

[5] A. La Rosa, et al., Proceedings of Science (RD09),Paper 032, 2010.

[6] E. Bolle, et al., Proceedings of Science (VERTEX 2009), Paper 016, 2010

[7] P. Hansson et al., Nucl. Instr. and Meth. A (2010), in press (doi:10.1016/j.nima.2010.06.321).

[8] FBK Fondazione Bruno Kessler, Trento, Italy. http://www.fbk.eu

[9] C. Kenney et al., IEEE Trans. Nucl. Sci. vol. 464 (1999) 1224.

[10] Stanford Nanofabrication Facility, weblink: http://snf.stanford.edu.

[11] Sintef, Oslo, Norway. http://www.sintef.no

[12] Centro National de Microelectronica, Barcelona, Spain. http://www.cnm.es/

[13] C. Da Via et al., Nucl. Instrum. Meth. A 603, (2009) 319.

[14] C. J. Kenney et al., IEEE Trans.Nucl. 48, (2001) 6.

[15] G.F. Dalla Betta et al., IEEE Nuclear Science Symposium, Knoxville, TN, USA, October 31-November 6, (2010), Paper N15-3.

[16] C. Piemonte et al., Nucl. Instrum. Meth. A 541, (2005) 441.

[17] G. Pellegrini et. al., Nucl. Instr. Meth. A487 (2002) 19.

[18] A. Zoboli et al., IEEE Trans. Nucl. Sci. 55, (2008) 2775

[19] I. Peric et al., Nucl. Instrum. Meth. A 565, (2006) 178.

[20] G. F. Dalla Betta et al., Nucl. Instrum. Meth. A, in press (doi:10.1016/j.nima.2010.04.079).

[21] D. Haas Proc of the LCWS2007, (2007), http://www.eudet.org

[22] G. Add (ATLAS Collaboration), JINST3, P07007, 7 (2008).

[23] G.F. Dalla Betta et al., Nucl. Instrum. Meth. A, in press (doi:10.1016/j.nima.2010.03.164).

[24] Forschungszentrum, Karlsruhe, Germany, http://www.fzk.de

[25] Jožef Stefan Institute, Lubiana, http://www.ijs.si/ijsw/JSI

[26] SELEX Sistemi Integrati, Roma, Italy, http://www.selex-si.com

[27] Fraunhofer IZM, Berlin, Germany. http://www.fraunhofer.de/en/

[28] V. Cindro et al., Nucl. Instrum. Meth. A 599, (2009) 60. 